\title{
Identification of undiagnosed type 2 diabetes by systolic blood pressure and waist-to-hip ratio
}

\author{
M. T. T. Ta $\cdot$ K. T. Nguyen $\cdot$ N. D. Nguyen $\cdot$ \\ L. V. Campbell • T. V. Nguyen
}

Received: 3 May 2010 / Accepted: 11 June 2010 /Published online: 2 July 2010

(C) Springer-Verlag 2010

\begin{abstract}
Aims/hypothesis We estimated the current prevalence of type 2 diabetes in the Vietnamese population and developed simple diagnostic models for identifying individuals at high risk of undiagnosed type 2 diabetes.

Methods The study was designed as a cross-sectional investigation with 721 men and 1,421 women, who were aged between 30 and 72 years and were randomly sampled from Ho Chi Minh City (formerly Saigon) in Vietnam. A $75 \mathrm{~g}$ oral glucose tolerance test to assess fasting and $2 \mathrm{~h}$ plasma glucose concentrations were determined for each individual. The ADA diagnostic criteria were used to determine the prevalence of type 2 diabetes. WHR and blood pressure were also measured in all individuals.
\end{abstract}

\section{T. T. Ta}

Department of Nutrition, Nhan dan Gia Dinh Hospital,

Ho Chi Minh City, Vietnam

\section{K. T. Nguyen}

Department of Endocrinology,

University of Medicine and Pharmacy,

Ho Chi Minh City, Vietnam

N. D. Nguyen $\cdot$ L. V. Campbell $\cdot$ T. V. Nguyen $(\bowtie)$

Garvan Institute of Medical Research,

384 Victoria Street, Darlinghurst,

Sydney, NSW 2010, Australia

e-mail: tuan.nguyen@unsw.edu.au

L. V. Campbell $\cdot$ T. V. Nguyen

St Vincent's Clinical School, University of New South Wales,

Sydney, NSW, Australia

T. V. Nguyen

School of Public Health and Community Medicine,

University of New South Wales,

Sydney, NSW, Australia
Results The prevalence of type 2 diabetes was $10.8 \%$ in men and $11.7 \%$ in women. Higher WHR and blood pressure were independently associated with a greater risk of type 2 diabetes. Compared with participants without central obesity and hypertension, the odds of diabetes was increased by 6.4 -fold (95\% CI 3.2-13.0) in men and 4.1fold (2.2-7.6) in women with central obesity and hypertension. Two nomograms were developed that help identify men and women at high risk of type 2 diabetes.

Conclusions/interpretation The current prevalence of type 2 diabetes in the Vietnamese population is high. Simple field measurements such as waist-to-hip ratio and systolic blood pressure can identify individuals at high risk of undiagnosed type 2 diabetes.

Keywords Blood pressure - Risk factors · Type 2 diabetes . Waist-to-hip ratio
Abbreviations
FPG Fasting plasma glucose
IFG Impaired fasting glucose
IGT Impaired glucose tolerance
NNS Number of individuals needed to undergo screening
ROC Receiver operating characteristic

\section{Introduction}

Type 2 diabetes mellitus is recognised as an important public health problem across the world, because it is highly prevalent in the general population and incurs serious health consequences $[1,2]$. The global prevalence of type 2 diabetes mellitus is projected to increase from $2.8 \%$ in 2000 to $4.4 \%$ in 2030, with the actual number of people with the disease doubling during the same period [3]. A large increase in the 
global prevalence of type 2 diabetes mellitus will take place in the developing world, with $80 \%$ of all new cases expected to appear in the developing countries by 2025 [4]. The AsiaPacific region, including Vietnam, has been identified as of 'prime importance to the epidemiology of diabetes' [5] because the prevalence of type 2 diabetes in this part of the world is higher than in other developing countries [1], possibly reflecting an even higher effect of evolutionary pressure and thrifty genes in some ethnic groups.

Few studies have examined the prevalence of type 2 diabetes and its risk factors in Vietnam [6, 7], where type 2 diabetes and obesity are increasingly becoming public health concerns. A difficult aspect of estimating the prevalence of type 2 diabetes is that the disease is often asymptomatic at onset and can remain undiagnosed for several years [7, 8], such that in the developed world approximately $50 \%$ of individuals with type 2 diabetes are undiagnosed [9]. However, universal screening is not cost-effective or recommended. The present study sought to (1) estimate the prevalence of, and risk factors for undiagnosed type 2 diabetes mellitus; and (2) develop nomograms for predicting type 2 diabetes risk in Vietnamese men and women.

\section{Methods}

Study design and participants The study was designed as a cross-sectional investigation, conducted in Ho Chi Minh City (formerly Saigon). The city is a major economic centre in Vietnam, with a population of 6.4 million and a population density of 3,400 people per square $\mathrm{km}$. The present study protocol and procedures were approved by the Ethics Committee of the Department of Science and Technology, Ho Chi Minh City.

Based on the electoral roll (in Vietnam voting is compulsory) of 17 districts within the city, we randomly selected 30 suburbs for sampling. The sampling scheme was based on the population proportionate to size methodology, in which for each selected suburb (cluster), the list of households and individuals aged between 30 and 70 years old was obtained. A computer program was then used to randomly select participants and send letters of invitation to participate in the study. The rate of acceptance was around $90 \%$. Participants received a free health check-up, but no reimbursement. Inclusion criteria were: men and women aged between 30 and 70 years, not known to have type 2 diabetes at time of acceptance to participate in the study. We excluded men or women who were deemed to have impaired cognitive function or were unwilling to give consent or were physically unable to complete the clinical examination.

Sample size was estimated based on the number of events per predictor in the logistic regression model. From the literature, we anticipated that there would be around five predictors of type 2 diabetes. According to Peduzzi et al. [10], the number of events per predictor should be 10 or more; as a result, we aimed to obtain at least 50 diabetic individuals for each sex in the study. Previous studies in Vietnam and Asia suggest that the prevalence of type 2 diabetes is about $10 \%$ [11]. Thus, the required sample size was estimated to be at least 500 for each sex. This sample size has an $80 \%$ chance of detecting an association between a risk factor and type 2 diabetes with an odds ratio of 1.5 or greater at a significance level of $5 \%$.

Clinical and biochemical measurements Anthropometric variables including age, weight and standing height were obtained. Body weight was measured using digital scales (HN-210; Omron, Tokyo, Japan) in indoor clothing without shoes. Height was determined without shoes on a portable stadiometer (Idea Height Scale 966; Hata Exercise Tool Company, Tokyo, Japan) with mandible plane parallel to the floor. BMI was calculated as weight $(\mathrm{kg})$ divided by the square of height (m). In addition, waist and hip circumference (and hence WHR) were also measured. Waist circumference was measured at the mid-way between the lower rib margin and the iliac crest, while hip circumference was measured at the level of the anterior superior iliac spine, where this could be felt, otherwise at the broadest circumference below the waist. The measurement was done by an unstretched tape meter to the nearest $0.1 \mathrm{~cm}$. The participants were instructed to wear light clothing and breathe gently during the measurements.

Body fat was measured using bioelectric impedance on a body fat analyser (BC-118; Tanita, Tokyo, Japan). Fat mass was expressed as per cent of body weight (i.e. per cent body fat).

Participants were instructed to avoid alcohol, cigarette smoking and coffee for at least $30 \mathrm{~min}$ before their blood pressure measurement. Three consecutive blood pressure measurements per participant were obtained by an attending doctor according to a standardised protocol, with participants in a seated position after having rested for at least $5 \mathrm{~min}$. A calibrated mercury sphygmomanometer was used, with one of four cuff sizes depending on the participant's arm circumference.

Diagnosis of type 2 diabetes All participants were instructed to fast overnight before the examination. Venous blood $(20 \mathrm{ml})$ was taken from each participant by venepuncture between 07:00 and 11:00 hours. The serum was immediately frozen to $-20^{\circ} \mathrm{C}$ prior to biochemical analysis. Fasting plasma glucose (FPG) level was measured by the glucose oxidase method (GOD-PAP; Boehringer-Mannheim, Mannheim, Germany). All specimens were analysed at the MEDIC's Department of Biochemistry and Paraclinical Services (Ho Chi Minh City, Vietnam). 
An OGTT was administered after an $8 \mathrm{~h}$ fast. A fasting blood sample for plasma glucose was obtained and then $75 \mathrm{~g}$ of monohydrate glucose solution was ingested. Blood samples were obtained at $120 \mathrm{~min}$ after ingestion and were later assayed for glucose concentrations.

Individuals were classified into five groups according to FPG and glucose tolerance limit as follows: (1) normal glucose tolerance if FPG was less than $5.5 \mathrm{mmol} / \mathrm{l}$ and $2 \mathrm{~h}$ plasma glucose was less than $7.8 \mathrm{mmol} / \mathrm{l}$; (2) isolated impaired fasting glucose (IFG) if FPG was between 5.5 and $6.9 \mathrm{mmol} / \mathrm{l}$, and/or $2 \mathrm{~h}$ plasma glucose was less than $7.8 \mathrm{mmol} / \mathrm{l}$; (3) isolated impaired glucose tolerance (IGT) if FPG was less than $5.5 \mathrm{mmol} / \mathrm{l}$ and $2 \mathrm{~h}$ plasma glucose was between 7.8 and $11.0 \mathrm{mmol} / \mathrm{l}$; (4) combined IFG and IGT if FPG was between 5.5 and $6.9 \mathrm{mmol} / \mathrm{l}$, and $2 \mathrm{~h}$ plasma glucose was between 7.8 and $11.0 \mathrm{mmol} / \mathrm{l}$; and (5) type 2 diabetes if FPG was equal to or higher than $7.0 \mathrm{mmol} / \mathrm{land} /$ or $2 \mathrm{~h}$ plasma glucose was $11.1 \mathrm{mmol} / \mathrm{l}$ or higher.

The study was approved by the Medical Ethics Committee of the Department of Health, Ho Chi Minh City, Vietnam. All volunteer participants were provided with full information about the study's purpose and gave informed consent to participate in the study, according to the principles of medical ethics of the World Health Organization.

Data analysis Our primary objective was to determine risk factors for undiagnosed type 2 diabetes and then develop a diagnostic model for predicting the risk of type 2 diabetes in an individual. The logistic regression model was used to develop a risk assessment model. The potential risk factors were age, weight, BMI, per cent body fat, abdominal fat, waist circumference, WHR, family history of diabetes, and systolic and diastolic blood pressure. Given multiple risk factors, many possible multiple logistic regression models could be formed to predict the risk of type 2 diabetes. In this study, we used the Bayesian model average approach [12] to search for a model with maximum parsimony (i.e. minimum number of risk factors and maximum discriminatory power). According to this approach, the model with the lowest Bayesian information criterion value is considered the most parsimonious. Based on the variable estimates from the most parsimonious model, nomograms [13] were constructed for prediction of the risk of type 2 diabetes. The prognostic performance of parsimonious models was assessed by the area under the receiver operating characteristic (ROC) curve $[14,15]$. The nomograms were internally validated by the bootstrap method, which assesses how accurately the model will predict type 2 diabetes in a new similar sample of participants. In this method, 500 sub-samples of the entire sample, each consisting of 150 participants, were repeatedly re-sampled (with replacement) and analysed, from which biases were estimated [16]. In a further validation, the predicted probability of type 2 diabetes was compared with the actual probability (i.e. nomogram calibration) in the entire sample, again using 1,000 bootstrap resample to reduce overfit bias, which would have overstated the accuracy of the nomogram. All analyses were performed using the R language [17] on a Windows XP platform.

\section{Results}

Baseline characteristics of participants Overall, approximately $49 \%$ men and 56\% women had impaired glucose homeostasis, with the majority being isolated IFG (men $23.5 \%$, women $22 \%$ ). The prevalence of type 2 diabetes in men (Fig. 1a) was $10.8 \%(78 / 721)$ and was not significantly different from women (Fig. 1b), among whom the prevalence was $11.7 \%(188 / 1,609)$.

Demographic, anthropometric and clinical characteristics of participants classified by diabetic status are shown in
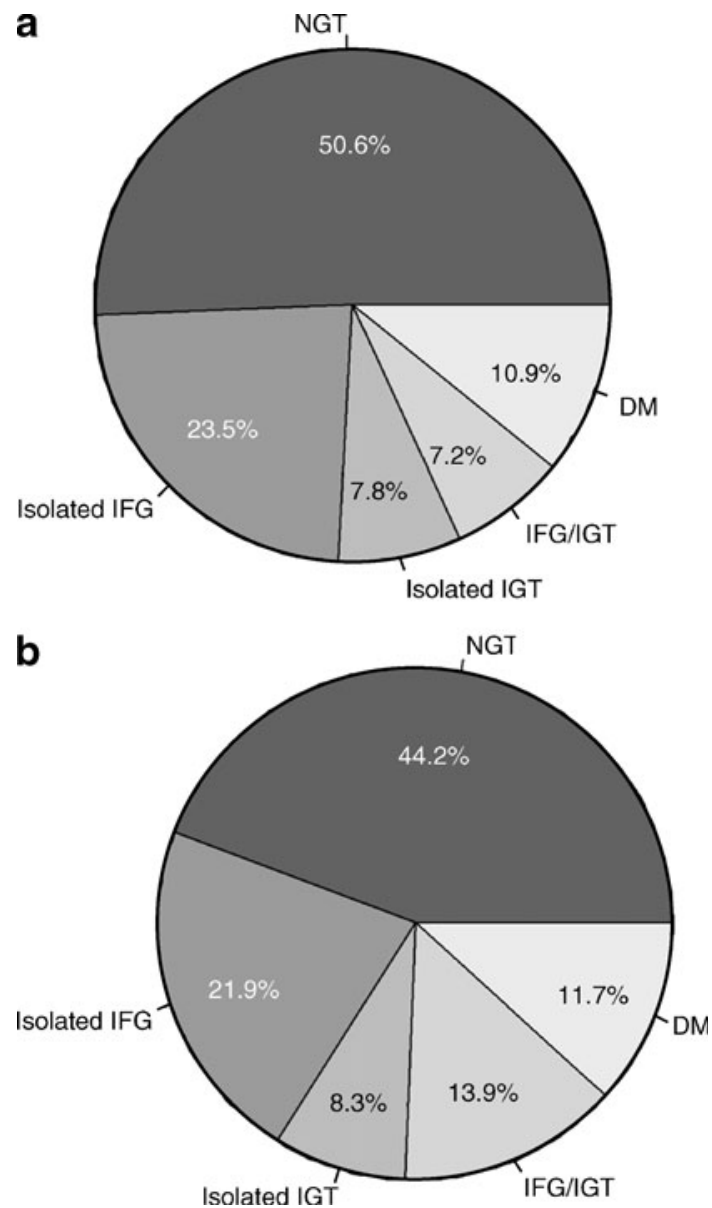

Fig. 1 Prevalence of impaired glucose homeostasis in Vietnamese men (a) and women (b) aged 30 years and above. DM, type 2 diabetes mellitus 
Table 1. The average age of participants was $~ 50$ (SD 6) years, with range being 34 to 72 years. In both sexes, there was no significant difference in age between those with and without type 2 diabetes.

As expected, men and women with type 2 diabetes had greater body weight, BMI, per cent body fat and abdominal fat than those without type 2 diabetes. Using the criterion of BMI $>25 \mathrm{~kg} / \mathrm{m}^{2}, 21.7 \%(n=157)$ men and $26.1 \%(n=420)$ women were classified as overweight or obese. However, in diabetic individuals, the prevalence of obesity was significantly higher than the population average. Approximately $40 \%(n=31)$ of diabetic men and $42 \%(n=79)$ of diabetic women had overweight or obesity.

In both sexes, diabetic individuals had significantly higher systolic and diastolic BP than non-diabetic individuals. For instance, systolic BP in diabetic men was on average $13 \mathrm{mmHg}$ higher than in non-diabetic men, a difference also observed in women $(9 \mathrm{mmHg})$. Among diabetic men, 55\% $(n=43)$ were hypertensive, which was significantly greater than among non-diabetic men, in whom prevalence of hypertension was $32 \%$. In women, hypertension was present in $38 \%$ of diabetic women, which was significantly higher than in non-diabetic women $(24 \%)$.

Risk factors and diagnostic model of type 2 diabetes Univariate analysis (Table 2) suggested that each of the risk factors in Table 1 was significantly associated with type 2 diabetes. In univariate analysis, WHR was the most important risk factor, with each 0.07 increase in WHR being associated with an odds ratio of 2.5 (95\% CI 1.85 $3.50)$ in women and $1.72(1.46-2.03)$ in men. Results of the Bayesian model average analysis suggested that blood pressure and measures of fat mass (e.g. weight, BMI, WHR, per cent body fat and abdominal fat) were related to type 2 diabetes.

Using the Bayesian information criteria as a metric of model selection, we found that the model with WHR and
Table 1 Baseline characteristics stratified by sex

\begin{tabular}{|c|c|c|c|}
\hline \multirow[t]{2}{*}{ Variable } & \multicolumn{2}{|l|}{ Diabetic status } & \multirow[t]{2}{*}{ Difference, mean $(95 \% \mathrm{CI}$} \\
\hline & Non-diabetes & Diabetes & \\
\hline \multicolumn{4}{|l|}{ Men } \\
\hline$n$ & 643 & 78 & \\
\hline Age (years) & $50.0(5.9)$ & $51.3(6.8)$ & $1.5(0.05,2.9)$ \\
\hline Weight (kg) & $58.9(10.3)$ & $64.2(10.8)$ & $5.2(2.8,7.7)$ \\
\hline BMI $\left(\mathrm{kg} / \mathrm{m}^{2}\right)$ & $22.2(3.75)$ & $24.2(3.7)$ & $2.0(1.1,2.8)$ \\
\hline Waist circumference $(\mathrm{cm})$ & $82.6(10.0)$ & $89.2(10.2)$ & $6.6(4.3,9.0)$ \\
\hline WHR & $0.90(0.06)$ & $0.95(0.06)$ & $0.05(0.03,0.06)$ \\
\hline Lean mass (kg) & $46.4(5.7)$ & $48.1(5.4)$ & $1.7(0.3,3.1)$ \\
\hline Per cent body fat & $20.4(6.4)$ & $25.7(7.0)$ & $3.7(2.1,5.2)$ \\
\hline Abdominal fat (kg) & $7.5(3.4)$ & $9.5(4.0)$ & $2.0(1.2,2.8)$ \\
\hline Systolic BP (mmHg) & $129(22)$ & $142(24)$ & $13(7,18)$ \\
\hline Diastolic BP (mmHg) & $83(14)$ & $88(12)$ & $5(2,8)$ \\
\hline Insulin $(\mathrm{pmol} / \mathrm{l})$ & $4.7(4.6)$ & $5.9(6.3)$ & $1.2(-0.05,2.46)$ \\
\hline \multicolumn{4}{|l|}{ Women } \\
\hline$n$ & 1,421 & 188 & \\
\hline Age (years) & $49.4(5.9)$ & $50.7(6.1)$ & $1.3(0.4,2.2)$ \\
\hline Weight $(\mathrm{kg})$ & $53.5(8.5)$ & $57.0(9.3)$ & $3.5(2.2,4.8)$ \\
\hline BMI $\left(\mathrm{kg} / \mathrm{m}^{2}\right)$ & $23.0(3.3)$ & $24.5(3.7)$ & $1.5(1.0,2.0)$ \\
\hline Waist circumference $(\mathrm{cm})$ & $84.9(9.8)$ & $89.5(9.8)$ & $4.5(3.0,6.0)$ \\
\hline WHR & $0.91(0.07)$ & $0.95(0.07)$ & $0.04(0.03,0.05)$ \\
\hline Lean mass (kg) & $34.3(5.9)$ & $36.7(6.4)$ & $2.4(1.5,3.3)$ \\
\hline Per cent body fat & $34.7(3.4)$ & $35.3(4.1)$ & $0.6(0.03,1.1)$ \\
\hline Abdominal fat (kg) & $10.7(3.6)$ & $12.4(3.8)$ & $1.7(1.1,2.2)$ \\
\hline Systolic BP (mmHg) & $124(20)$ & $133(21)$ & $9(6,12)$ \\
\hline Diastolic BP (mmHg) & $78(12)$ & $82(12)$ & $4(2,6)$ \\
\hline Insulin (pmol/1) & $5.2(4.3)$ & $6.8(5.1)$ & $1.5(0.8,2.3)$ \\
\hline
\end{tabular}

Unless otherwise indicated, values are mean (SD)

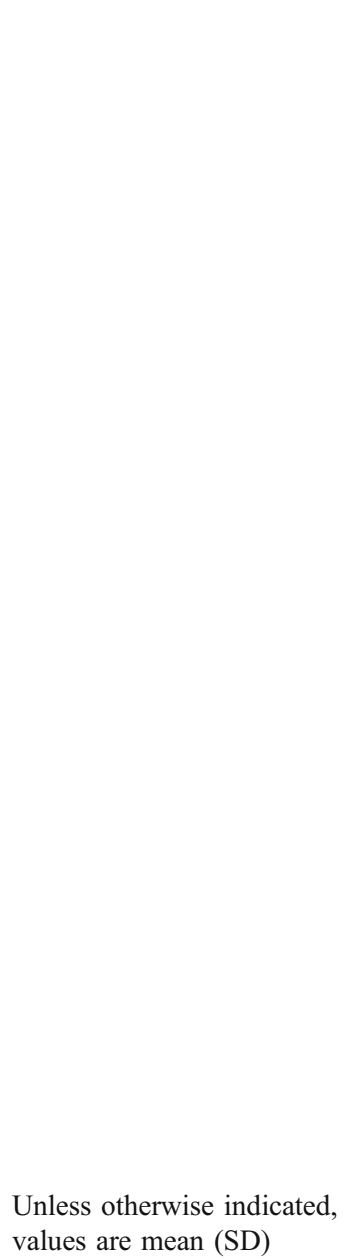


Table 2 Association between risk factor and type 2 diabetes: univariate logistic regression analysis

\begin{tabular}{|c|c|c|c|c|c|}
\hline \multirow[t]{2}{*}{ Risk factor } & \multirow[t]{2}{*}{ Comparison unit ${ }^{\mathrm{a}}$} & \multicolumn{2}{|l|}{ Men } & \multicolumn{2}{|l|}{ Women } \\
\hline & & OR $(95 \% \mathrm{CI})$ & c statistic & OR $(95 \% \mathrm{CI})$ & c statistic \\
\hline Age (years) & 5 & $1.28(1.05-1.56)$ & 0.58 & $1.19(1.05-1.36)$ & 0.56 \\
\hline Weight (kg) & 10 & $1.57(1.26-1.96)$ & 0.64 & $1.53(1.30-1.81)$ & 0.61 \\
\hline Waist circumference $(\mathrm{cm})$ & 10 & $1.89(1.48-2.40)$ & 0.69 & $1.60(1.37-1.86)$ & 0.63 \\
\hline WHR & 0.07 & $2.54(1.85-3.50)$ & 0.71 & $1.72(1.46-2.03)$ & 0.64 \\
\hline Lean mass $(\mathrm{kg})$ & 7 & $1.46(1.08-1.96)$ & 0.59 & $1.36(1.00-1.85)$ & 0.55 \\
\hline Fat mass (kg) & 7 & $1.84(1.43-2.38)$ & 0.66 & $1.60(1.36-1.88)$ & 0.62 \\
\hline Per cent body fat & 10 & $2.29(1.61-3.28)$ & 0.66 & $2.01(1.54-2.65)$ & 0.62 \\
\hline Abdominal fat (kg) & 4 & $1.77(1.38-2.27)$ & 0.65 & $1.58(1.35-1.84)$ & 0.63 \\
\hline Systolic BP (mmHg) & 20 & $1.62(1.32-2.00)$ & 0.65 & $1.50(1.31-1.73)$ & 0.63 \\
\hline Diastolic BP (mmHg) & 12 & $1.44(1.16-1.79)$ & 0.62 & $1.40(1.21-1.61)$ & 0.61 \\
\hline
\end{tabular}

${ }^{\text {a }}$ The comparison unit was set to be close to the standard deviation of each risk factor

systolic BP was the most parsimonious. In this model, each SD (0.07) lower in WHR was associated with a 2.26-fold (1.6-3.1) and 1.59-fold (1.35-1.88) increase in the odds of type 2 diabetes in men and women, respectively. The odds ratio for systolic BP (per $20 \mathrm{mmHg}$ increase) was 1.41 (1.13-1.76) in men and $1.52(1.29-1.58)$ in women. The magnitude of association between these risk factors and type 2 diabetes was somewhat lower in women than in men. The area under the ROC curve for this model was 0.73 for men and 0.67 for women. Internal validation by the bootstrap method suggested that the maximum calibration error in predicting probability of type diabetes was about $0.6 \%$ in men and $0.4 \%$ in women. The predicted risk was slightly overestimated in the highest risk group compared with observed risk in men, but the opposite applied in women (data not shown).

Based on the model's variables, we estimated the risk of type 2 diabetes in men (Fig. 2a) and women (Fig. 2b) according to the following equations: risk $=1 /\left(1+\mathrm{e}^{x}\right)$, where $x=-15.194+11.615 \times \mathrm{WHR}+0.017 \times \mathrm{SBP}$ for men, and $x=-10.297+6.669 \times \mathrm{WHR}+0.016 \times \mathrm{SBP}$ for women (SBP denotes systolic blood pressure). Since the magnitude of the association between each risk factor and diabetic risk was greater in men than in women, the predicted risk of type 2 diabetes in men was higher than in women for a given WHR and systolic BP.

We next addressed the question of how many cases of type 2 diabetes could be attributed to excess abdominal fat (as estimated by WHR) and/or hypertension. Based on the distribution of risk factors and their associated risk ratio, $66.5 \%$ (95\% CI 47.7-85.4\%) and 56\% (95\% CI 34.1$78.6 \%$ ) of type 2 diabetes in men and women, respectively, could be attributed to blood pressure and WHR. A majority of this attributable fraction was due to WHR. After adjusting for systolic blood pressure, 51.4\% (95\% CI
$30.5-72.4 \%)$ and $43.5 \%$ (95\% CI 17.5-69.4\%) in men and women, respectively, could be attributed to WHR.

Predictive nomograms Using the estimated variables in the final model, we constructed two nomograms for predicting
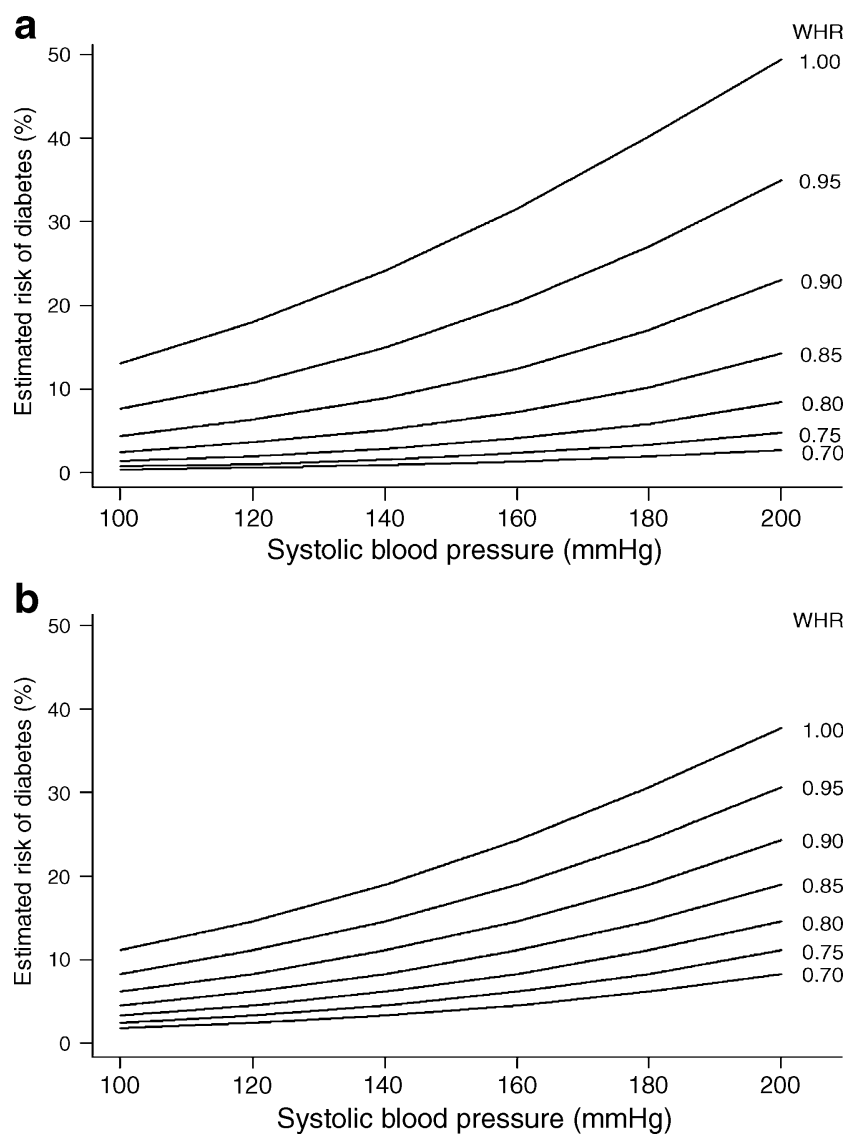

Fig. 2 Predicted risk of type 2 diabetes in men (a) and women (b) for a given systolic blood pressure and WHR 


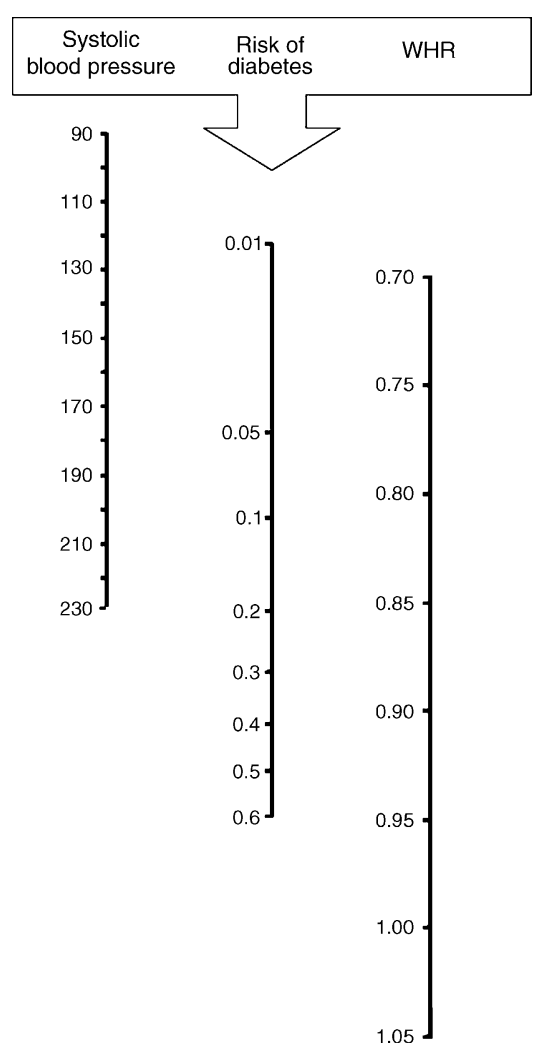

Fig. 3 Nomogram for predicting the risk of type 2 diabetes for a man. The nomogram has two columns representing WHR and systolic BP, with a vertical line between them that indicates the predicted risk of diabetes. For a man with systolic BP of $140 \mathrm{mmHg}$ and WHR 0.95, simply draw a straight line from the systolic BP axis at 140 to the WHR axis at 0.95 . The point of intersection between the straight line and the middle axis is the predicted probability of diabetes (here $\sim 15 \%$ )

the risk of type 2 diabetes in men (Fig. 3) and women (Fig. 4) separately. Each nomogram has two axes representing WHR and systolic BP, with a vertical line between them indicating the predicted risk of type 2 diabetes. Clinical applications of the nomograms can be illustrated by the following case scenario of a man with systolic BP $140 \mathrm{mmHg}$ and WHR 0.95 . On Fig. 3, a straight line drawn from the systolic BP axis to the WHR axis intersects the risk of diabetes axis at the point that predicts probability of type 2 diabetes (i.e. $~ 15 \%$ ).

\section{Discussion}

The growing prevalence of type 2 diabetes and cardiovascular diseases in developing countries is a global concern [18], but there are few data on risk factors of type 2 diabetes in these countries. In this study, we have shown that in asymptomatic individuals of Southeast Asian background aged between 30 and 70 years, the prevalence of type 2 diabetes was as high as $11 \%$ in men and $12 \%$ in women. These prevalence rates are even higher than in developed countries, where prevalence ranges between $6 \%[19]$ and $8 \%$ [20]. We have also shown that, using simple field measures such as blood pressure and WHR, it is possible to identify a substantial number of high-risk individuals.

Both risk factors have a biological basis. Hypertension has long been known to be a consequence of type 2 diabetes, but recent studies suggest that it may also precede the disease $[21,22]$. Excessive body fat, especially central fat, has long been recognised as a risk factor for type 2 diabetes. Our data show that central adiposity was associated with 3.7fold and 2.4-fold increases in the risk of type 2 diabetes, findings that are very comparable to those of a prospective study, where central obesity was associated with a 2.8 -fold increase in the risk of developing type 2 diabetes [23]. Taken together, the results of this study are consistent with that presumption and support the view that systolic blood pressure and WHR can be used as simple and robust risk factors to identify individuals at high risk of type 2 diabetes.

A number of prognostic models have been developed for predicting the risk of type 2 diabetes in Whites [24-28] and Asians [29]. These models use risk factors such as ethnicity, age, sex, family history of diabetes, indicators of hypertension (e.g. systolic BP, use of anti-hypertensive drugs) and measures of obesity (e.g. BMI, waist circumference). The

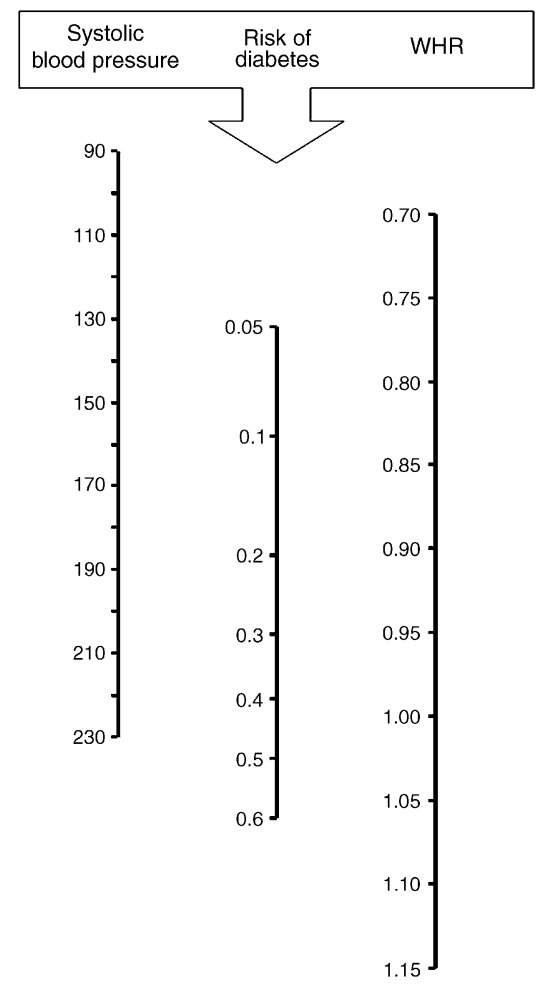

Fig. 4 Nomogram for predicting the risk of type 2 diabetes for a woman. The nomogram has two axes representing WHR and systolic $\mathrm{BP}$, with a vertical line between them that indicates the predicted risk of diabetes. Using the method described (Fig. 3) and identical values, the predicted probability of diabetes for a woman is $\sim 13 \%$ 
area under the ROC curve of these models varies between 0.54 and 0.83 . In this study, we found only two risk factors, systolic blood pressure and WHR, that are significant predictors of type 2 diabetes risk; the area under the ROC curve model with the two risk factors was 0.73 for men and 0.69 for women. Thus, the prognostic performance of our model is comparable to that of previous models with more risk variables. However, there are several differences between our model and previous ones. Most previous models, such as that of Gao et al. [29], are based on the concept of risk stratification, in which continuous variables are categorised into subgroups. Our model, in contrast, is based on the concept of individualisation, with the continuous nature of risk factors being preserved in order to increase the degree of uniqueness of an individual. With continuous variables, the more risk factors that are considered, the greater the likelihood that the uniqueness of an individual's profile can be defined. Therefore, by modelling risk factors on their continuous scale, the models of the present study can be uniquely tailored to an individual.

Recent analysis suggests that screening for type 2 diabetes could be cost-effective in white populations [30]. Although such an analysis has not been done in Asian populations, assessment of absolute risk of type 2 diabetes can have practical applications in treatment allocation, risk communication and decision-making. Not all individuals with high predicted risk of type 2 diabetes actually have diabetes; conversely, not all individuals with low predicted risk of diabetes are free of the disease. Ideally, the nomograms presented here are best used as an initial screening tool followed by confirmatory or diagnostic tests, including assessment of glycaemia, $\mathrm{HbA}_{1 \mathrm{c}}$, lipids and family history. To this end, it is important to derive a threshold for decision making. Such a threshold is a function of test sensitivity, test specificity, cost-effectiveness considerations and risks of treatment [31], the latter of which are not currently available. Therefore, further research is required to derive these thresholds for the assessment of diabetic risk. While such a threshold remains to be determined, it is possible to consider the number of individuals needed to undergo screening (NNS) and the ensuing diagnosis or intervention that would be needed to reduce cases with adverse outcome by one. Assuming that an intervention reduces the risk of diabetes by $30 \%$ if the threshold is set at $15 \%$, then the NNS would range between 65 (for men) and 68 (for women); if the threshold was set at $10 \%$, the NNS would fall to 41 men and 43 women. Thus, the present nomogram may help in selective screening for type 2 diabetes and save on universal non-selective screening test.

The present findings should be interpreted within the context of strengths and potential weaknesses. A major strength of this study is that the diagnosis of type 2 diabetes mellitus was by OGTT incorporating FPG, which mini- mises any possibility of misdiagnosis. The sample size was large enough to ensure that the study could assess modest effect sizes otherwise not possible in smaller studies. The participants were randomly selected from the general population by a rigorous sampling methodology, which enhances the external validity of this study. However, as the study was undertaken in an Asian city setting, the models derived from this study may not be applicable to rural populations or non-Asian populations, whose lifestyle and demographic structure may be different from those of the present population. Indeed, clinical diagnostic models of diabetes can perform well in one population, but not in other populations [25]. The age range of participants in this study was relatively narrow (mostly between 40 and 60 years), which could also limit the generalisability of the present findings to the general population. Nevertheless, individuals in this age range are ideal for screening because, compared with younger working adults, they represent the economically productive members of the general population with a relatively short time to type 2 diabetes development.

In summary, these data show an already high prevalence of type 2 diabetes in an urban Vietnamese population, namely $11 \%$ in men and $12 \%$ in women. They also show that systolic blood pressure and WHR can be used for identifying individuals at high risk of undiagnosed type 2 diabetes. Our findings thus suggest that measurement of simple clinical indices (i.e. WHR and blood pressure) available to all health workers could account for half of the diabetic cases detected.

Acknowledgements The study was supported by a grant from the Department of Science and Technology, Ho Chi Minh City. We thank all participants for their enthusiasm and cooperation in the study.

Duality of interest N. D. Nguyen is supported by a grant from the Australian Medical Bioinformatics Resource (AMBeR). T. V. Nguyen is supported by a senior research fellowship from the Australian National Health and Medical Research Council. All other authors declare there is no duality of interest associated with this manuscript.

\section{References}

1. King H (1999) First International Forum for Diabetes Outcome Research. Copenhagen: Denmark International Diabetes Monitor, April 22-23

2. Must A, Spadano J, Coakley EH, Field AE, Colditz G, Dietz WH (1999) The disease burden associated with overweight and obesity. JAMA 282:1523-1529

3. Wild S, Roglic G, Green A, Sicree R, King H (2004) Global prevalence of diabetes: estimates for the year 2000 and projections for 2030. Diab Care 27:1047-1053

4. Shaw JE, Chisholm DJ (2003) 1: Epidemiology and prevention of type 2 diabetes and the metabolic syndrome. Med J Aust 179:379-383

5. Cockram CS (2000) The epidemiology of diabetes mellitus in the Asia-Pacific region. Hong Kong Med J 6:43-52 
6. Quoc PS, Charles MA, Cuong NH et al (1994) Blood glucose distribution and prevalence of diabetes in Hanoi (Vietnam). Am J Epidemiol 139:713-722

7. Duc Son LN, Kusama K, Hung NT et al (2004) Prevalence and risk factors for diabetes in Ho Chi Minh City, Vietnam. Diabet Med 21:371-376

8. No authors listed (2004) Screening for type 2 diabetes. Diab Care 27(Suppl 1):S11-S14

9. Harris MI, Flegal KM, Cowie CC et al (1998) Prevalence of diabetes, impaired fasting glucose, and impaired glucose tolerance in U.S. adults. The Third National Health and Nutrition Examination Survey, 1988-1994. Diab Care 21:518-524

10. Peduzzi P, Concato J, Kemper E, Holford TR, Feinstein AR (1996) A simulation study of the number of events per variable in logistic regression analysis. J Clin Epidemiol 49:1373-1379

11. Wen CP, David Cheng TY, Tsai SP, et al. (2008) Are Asians at greater mortality risks for being overweight than Caucasians? Redefining obesity for Asians. Public Health Nutr: 1-10

12. Hoeting J (1999) Bayesian model averaging: a tutorial. Stat Sci $14: 382-417$

13. Harrel FE (2001) Regression modeling strategies with applications to linear models, logistic regression, and survival analysis. Springer, New York

14. Hanley JA, McNeil BJ (1983) A method of comparing the areas under receiver operating characteristic curves derived from the same cases. Radiology 148:839-843

15. Swets JA (1986) Indices of discrimination or diagnostic accuracy: their ROCs and implied models. Psychol Bull 99:100-117

16. Harrell FE Jr, Lee KL, Mark DB (1996) Multivariable prognostic models: issues in developing models, evaluating assumptions and adequacy, and measuring and reducing errors. Stat Med 15:361-387

17. R Development Core Team (2008) R: A language and environment for statistical computing. R Foundation for Statistical Computing, Vienna. Available from www.R-project.org

18. Hossain P, Kawar B, El Nahas M (2007) Obesity and diabetes in the developing world - a growing challenge. N Engl J Med 356:213-215

19. No authors listed (2003) Prevalence of diabetes and impaired fasting glucose in adults-United States, 1999-2000. JAMA 290:1702-1703
20. Dunstan DW, Zimmet PZ, Welborn TA et al (2002) The rising prevalence of diabetes and impaired glucose tolerance: the Australian Diabetes, Obesity and Lifestyle Study. Diab Care 25:829-834

21. Conen D, Ridker PM, Mora S, Buring JE, Glynn RJ (2007) Blood pressure and risk of developing type 2 diabetes mellitus: the Women's Health Study. Eur Heart J 28:2937-2943

22. Flaa A, Sandvik L, Kjeldsen SE, Eide IK, Rostrup M (2008) Does sympathoadrenal activity predict changes in body fat? An 18-y follow-up study. Am J Clin Nutr 87:1596-1601

23. Ohnishi H, Saitoh S, Takagi S et al (2006) Incidence of type 2 diabetes in individuals with central obesity in a rural Japanese population: the Tanno and Sobetsu study. Diab Care 29:11281129

24. Glumer C, Carstensen B, Sandbaek A, Lauritzen T, Jorgensen T, Borch-Johnsen K (2004) A Danish diabetes risk score for targeted screening: the Inter99 study. Diab Care 27:727-733

25. Glumer C, Vistisen D, Borch-Johnsen K, Colagiuri S (2006) Risk scores for type 2 diabetes can be applied in some populations but not all. Diab Care 29:410-414

26. Hippisley-Cox J, Coupland C, Robson J, Sheikh A, Brindle P (2009) Predicting risk of type 2 diabetes in England and Wales: prospective derivation and validation of QDScore. BMJ 338: b880

27. Schmidt MI, Duncan BB, Bang $H$ et al (2005) Identifying individuals at high risk for diabetes: the Atherosclerosis Risk in Communities study. Diab Care 28:2013-2018

28. Wilson PW, Meigs JB, Sullivan L, Fox CS, Nathan DM, D'Agostino RB Sr (2007) Prediction of incident diabetes mellitus in middle-aged adults: the Framingham Offspring Study. Arch Intern Med 167:1068-1074

29. Gao WG, Dong YH, Pang ZC et al (2010) A simple Chinese risk score for undiagnosed diabetes. Diabet Med 27:274-281

30. Kahn R, Alperin P, Eddy D et al (2010) Age at initiation and frequency of screening to detect type 2 diabetes: a cost-effectiveness analysis. Lancet 375:1365-1374

31. Pauker SG, Kassirer JP (1980) The threshold approach to clinical decision making. N Engl J Med 302:1109-1117 